\title{
Patrones de comportamiento de la calidad del agua de ecosistemas hídricos y su relación con la ocupación del territorio en el Chocó Biogeográfico
}

\section{Behavior patterns of the water quality of water ecosystems and their relationship with the occupation of the territory in the Chocó Biogeográfico}

\section{Lady Vargas Porras*}

\section{Resumen}

Con los datos obtenidos en caracterizaciones ambientales realizadas en 12 cuerpos de agua distribuidos altitudinalmente en la región del Chocó Biogeográfico, se aplicó un análisis de componentes principales (PCA) y gráficos de dispersión, que permitieron establecer un patrón de comportamiento de la calidad del agua y evaluar la relación entre variables fisicoquimicas, biológicas y sociales. El PCA arrojó 5 componentes que explicaron el 90\% de la variabilidad de los datos. Tres de estos componentes indicaron fuertes relaciones entre la concentración de nutrientes y el número de individuos de macroinvertebrados, además, de la altitud, la conductividad, el pHy la presencia de vertimientos mineros y desechos antrópicos, así como también entre el nivel poblacional y la concentración de coliformes fecales, grasas y aceites. Los resultados permitieron concluir que el patrón de comportamiento de la calidad de agua en la región está determinado no solo por el nivel poblacional presente en el área de influencia de las fuentes hídricas, sino por una combinación de elementos que incluyen el tipo de prácticas productivas desarrolladas, el estado de los sistemas de saneamiento básico y la distribución altitudinal de la ocupación del territorio que es una característica cultural de la región.

Palabras clave: Calidad del agua, Características fisicoquímicas de ríos, Fuentes hídricas superficiales, Macroinvertebrados acuáticos.

\section{Abstract}

With the data obtained in environmental characterizations performed in 12 basins distributed altitudinally in the Chocó Biogeographic region, a principal component analysis (PCA) and dispersion charts were applied, which allowed to establish a pattern behaviour of the quality of the water and to evaluate the

\footnotetext{
Ingeniera ambiental, Ms (C) Ciencias Ambientales, Investigadora Principal, Instituto de Investigaciones Ambientales del Pacífico (IIAP), Quibdó, Colombia. e-mail: 1vargas@,iiap.org.co
} 
the relationship between physicochemical variables, biological and social. The PCA yielded 5 components that explained $90 \%$ of the variability of the data. Three of these components indicated strong relationships between nutrient concentration and the number of macroinvertebrate individuals, in addition to altitude, conductivity, $\mathrm{pH}$ and the presence of mineral spills and anthropic wastes, as well as between population level and concentration fecal coliforms, fats and oils. The results allowed to conclude that the pattern of behavior of the water quality in the region is determined not only by the population level present in the area of influence of the water sources, but by a combination of elements that include the type of productive practices developed, the state of the basic sanitation systems and the altitudinal distribution of the occupation of the territory that is a cultural characteristic of the region.

Keywords: Aquatic macroinvertebrates, Physicochemical characteristics of rivers, Surface water sources, Water quality.

\section{Introducción}

El incremento y la concentración de poblaciones humanas alrededor de las fuentes hídricas, traen consigo afectaciones sobre el recurso, como consecuencia del desarrollo de diferentes actividades y malas prácticas que facilitan la incorporación de contaminantes a los cuerpos de agua, entre ellas: insuficiente planificación de las actividades urbanas e industriales, uso inadecuado de los suelos, deforestación y manejo inadecuado de desechos domiciliarios, agropecuarios e industriales (Coto, 1998). Así, la calidad de aguas superficiales es un tema de discusión en todo el mundo, fundamentalmente porque se ve afectada con la introducción de agentes contaminantes de origen antrópico, cada vez más agresivos y que por su naturaleza química son más difíciles de tratar. Además, el cambio de uso de suelo influ- ye significativamente sobre el paisaje alterando ecosistemas y recursos naturales (Rodríguez et al., 2002; Álvarez et al., 2008)

La región del Chocó Biogeográfico está habitada por comunidades afrodescendientes e indígenas con creencias y costumbres muy propias que manifestan a través de su cultura y sus conocimientos ancestrales, lo que ha determinado su relación con el entorno y las dinámicas de uso y aprovechamiento de los recursos naturales, siendo el agua uno de los más importantes para su desarrollo. El área concentra además una gran riqueza hídrica distribuida altitudinalmente en diferentes tipos de ecosistemas, donde el nivel de intervención y la dinámica de uso depende de criterios como la ubicación, la facilidad del acceso, las condiciones climáticas y en gran medida las características socioculturales de las poblaciones que habitan la región, criterios que en conjunto determinan las formas de uso del recurso y a su vez el estado de conservación de los mismos, por la relación existente entre el hombre y el entorno.

En este sentido, criterios socioculturales como las formas de ocupación del territorio y las condiciones de saneamiento básico han influido en el estado y la calidad del recurso hídrico en la región, haciendo que las zonas con mayor concentración de población correspondan con los sitios con mayores índices de contaminación del agua, dada la baja cobertura de servicios públicos como acueducto, alcantarillado y aseo, que presentan un gran rezago en relación con el resto del país, porque en la mayoría de los municipios hay carencia de la infraestructura o se requiere ampliación o adecuación para lograr mejorar la atención a las comunidades y con ello favorecer la protección de los cuerpos de agua. Esto en contraste con las áreas que por costumbres y características culturales no son habitadas o son usadas solo para recolección de recursos del bosque haciendo que se mantengan los altos niveles de conservación ecosistémica y excelente calidad de agua en las fuentes hídricas asociadas. 


\section{Bioetnia Volumen 12, 2015}

Desde esta perspectiva, el presente trabajo busca analizar los cambios de calidad que se presentan en los cuerpos de agua superficiales distribuidos en el gradiente altitudinal de la región del Chocó Biogeográfico, partiendo de resultados obtenidos en diferentes trabajos realizados por el Instituto de Investigaciones Ambientales del Pacífico (IIAP), Quibdó, Colombia. Los análisis se presentan desde la relación entre fuentes hídricas, las condiciones ambientales de los ecosistemas que los albergan y las características socioculturales de las comunidades que se ubican en sus áreas de influencia.

Área de estudio. Estuvo conformada por 12 fuentes hídricas, distribuidas altitudinal y geográficamente en la región del Chocó Biogeográfico, las cuales se describen a continuación: En el río Cucurrupi, se obtuvieron reportes de la zona de nacimiento del mismo el cual se encuentra en las coordenadas 404'19.6" $\mathrm{N}$ y $76^{0} 30^{\prime} 30.5^{\prime}$ 'W, a $3.514 \mathrm{msnm}$ y en la zona aguas abajo del río perteneciente a la vertiente del río San juan en las coordenadas 404'26.72" N y 76030'28.9" W.

En el río Copomá, los muestreos se ubicaron muy cerca del nacimiento del mismo en las coordenadas $4^{0} 4^{\prime} 25^{\prime}$ 'N y $76^{0} 30^{\prime} 47^{\prime}$ 'W a una altura de $3.424 \mathrm{msnm}$. En las zonas anteriores de muestreo, las variaciones de temperatura oscilan entre $11^{\circ} \mathrm{C}$ y $15^{\circ} \mathrm{C}$ y $7^{\circ} \mathrm{C}$ y $15^{\circ} \mathrm{C}$; además, las condiciones climáticas del municipio del Litoral del San Juan son similares en gran parte del territorio, presentándose temperaturas promedio anuales que oscilan entre $\operatorname{los} 23^{\circ} \mathrm{C}$ y $28^{\circ} \mathrm{C}$, con un alto grado de humedad. En cuanto a su población, en esta zona se encuentran pocos grupos étnicos $\mathrm{y}$ en especial indígenas debido a que la mayor parte de los habitantes son de tierras bajas, lo que trae consigo la poca realización de actividades domésticas o antrópicas en el área.

Los reportes obtenidos del río Ingara, corresponde a la zona ubicada en las coordenadas $5^{\circ} 7.26 .2^{\prime \prime}$ y $76^{\circ} 4.0^{\prime} 12.4^{\prime \prime}$, a una altura de 3.513 msnm, y presenta valores de temperatura que oscilan entre $\operatorname{los} 4^{\circ} \mathrm{C}$ y $22^{\circ} \mathrm{C}$; en este, se trabajaron varios tramos a lo largo del río y al igual que los anteriores, en la parte alta y media del mismo, se encuentran pocos asentamientos humanos, por lo que se genera una disminución en el desarrollo de diferentes actividades alrededor de la fuente.

La quebrada Chocolate, se encuentra ubicada en la coordenadas $6^{\circ} 42^{\prime} 10.5^{\prime \prime} \mathrm{N}-76^{\circ} 33^{\prime} 23.4^{\prime \prime} \mathrm{W}$, a una altura de $993 \mathrm{msnm}$. En esta se trabajaron las zonas correspondientes a su nacimiento, la zona media y aguas debajo de la comunidad; en relación con su climatología, en el municipio de Frontino, los valores mínimos de temperatura fluctúan entre $12^{\circ} \mathrm{C}$ y $14^{\circ} \mathrm{C}$, los medios entre $20^{\circ} \mathrm{C}$ y $22^{\circ} \mathrm{C}$ y los máximos entre $26^{\circ} \mathrm{C}$ y $31^{\circ} \mathrm{C}$. Dentro del área de influencia, se identifica la población tradicional de indígenas Embera eyabidá, quienes tienen como sus principales actividades la tumba del monte, la preparación de colinos para la siembra del plátano y la cacería.

El río Tutunendo se encuentra ubicado en las coordenadas $5^{\circ} 44^{\prime} 31.1$ 'N y $76^{\circ} 31^{\prime} 192$ ' $\mathrm{W}$, a una altura de $50 \mathrm{msnm}$ y un valor promedio de temperatura de $26^{\circ} \mathrm{C}$ para el municipio; en la cuenca del río Tutunendo, se trabajó la zona alta, media y baja del mismo, identificando en su zona media la mayor parte de la población del municipio de Tutunendo, las cuales desarrollan actividades domésticas sobre la fuente, y en la zona baja el desarrollo de actividades mineras en el cuerpo de agua.

La quebrada Chinchano tributaria del río Timbiqui, se encuentra ubicada en las coordenadas $2^{\circ} 46^{\prime} 31.1$ " $\mathrm{N}$ y $77^{\circ} 40.1^{\prime} 42.2^{\prime \prime} \mathrm{W}$, a una altura de $5 \mathrm{msnm}$. El municipio cuenta con comunidades negras e indígena, que realizan actividades como la agricultura, la pesca, aprovechamiento forestal y explotación minera, lo que trae como consecuencia alteraciones sobre el recurso hídrico. Por su parte, la quebrada Agua Dulce, afluente del río Bubuey, se localiza en las coordenadas $\mathrm{N}$ $2^{\circ} 48^{\prime} 0.9^{\prime \prime} \mathrm{N}$ y W $77^{\circ} 38^{\prime} 59.3^{\prime}$ ' $\mathrm{W}$, a una altura de $5 \mathrm{msnm}$.

Para el caso del río Quito, se tuvo en cuenta la 
parte media y baja del mismo, las cuales se ubican en las coordenadas $5^{\circ} 25^{\prime} \mathrm{N}, 76^{\circ} 40^{\prime} \mathrm{W}$ y $5^{\circ} 38^{\prime}$ $33.9^{\prime}$ N, $76^{\circ} 43^{\prime} 18.2 \mathrm{~W}$ respectivamente, a una altura de $45 \mathrm{msnm}$. El municipio se caracteriza por presentar temperaturas mayores a $24^{\circ} \mathrm{C}$ y precipitaciones desde 8.000 a $10.000 \mathrm{~mm}$; este posee diferentes grupos étnicos especialmente afrodescendientes, quienes en conjunto con personas foráneas procedentes de diferentes partes del país, realizan actividades mineras sobre el cauce del río alterando no solo sus condiciones fisicoquímicas, sino también las condiciones naturales del sistema hídrico.

En el río Jella y la Quebrada Huina, se trabajaron las intersecciones entre estos y la bahía de ciudad Mutis, las cuales se localizan en las coordenadas $6^{\circ} 13^{\prime} 45.2^{\prime \prime} \mathrm{N}, 77^{\circ} 23^{\prime} 55.9^{\prime \prime} \mathrm{W}$ y $6^{\circ} 16^{\prime} 16.6^{\prime \prime} \mathrm{N}$, $77^{\circ} 27^{\prime} 25.9^{\prime \prime} \mathrm{W}$ respectivamente. El municipio presenta temperaturas promedios de $26.3^{\circ} \mathrm{C}$ y precipitaciones promedio anuales superiores a los $5.000 \mathrm{~mm}$; alrededor de esta fuente sus poblaciones realizan diferentes actividades entre estas domésticas, las cuales son vertidas al cuerpo de agua.

En el río Dagua, se tuvo en cuenta la zona de intersección entre este y la bahía de Buenaventura, la cual se encuentra en las coordenadas $6^{\circ} 13^{\prime} 45.2^{\prime}$ N y $77^{\circ} 23^{\prime} 55.9^{\prime \prime} \mathrm{W}$. El municipio presenta temperaturas de $27^{\circ} \mathrm{C}$ y sus poblaciones realizan actividades domésticas que vierten a la bahía y traen consigo contaminantes que alteran las condiciones fisicoquímicas del recurso.

Finalmente, se trabajó la intersección entre el caño Waffe y la bahía de Turbo, la cual se encuentra en las coordenadas $8^{\circ} 5^{1} 23.2^{11} \mathrm{~N}$ y $76^{\circ}$ $43^{1} 57.0^{11} \mathrm{~W}$. El municipio de Turbo, presenta temperaturas promedio de $27^{\circ} \mathrm{C}$ y precipitaciones de $2.426 \mathrm{~mm}$ al año. Sus diferentes grupos étnicos afrocolombianos, mestizos, blancos e indígenas realizan actividades antrópicas y domésticas que son vertidas al caño, además de los vertimientos líquidos provenientes del alcantarillado y los productos oleosos originados por el transporte de embarcaciones en la zona.

\section{Métodos}

Para analizar los patrones de comportamiento de la calidad fisicoquímica y biológica del agua en ecosistemas hídricos y su relación con la ocupación del territorio en la región del Chocó Biogeográfico, se evaluaron los resultados de parámetros fisicoquímicos y biológicos obtenidos en caracterizaciones ambientales realizadas en 12 cuerpos de agua distribuidos altitudinal y geográficamente en la región, abarcando desde zonas de alta y media montaña, bosque premontanos, selva pluvial y zonas costeras. El análisis incluyó las variables fisicoquímicas de oxígeno disuelto (OD), $\mathrm{pH}$, conductividad, turbiedad, sulfatos, nitratos, nitritos, fosfatos, coliformes fecales, grasas y aceites, así como número de individuos, familias y géneros de macroinvertebrados colectados en cada cuerpo de agua. Se realizó un análisis descriptivo de cada variable y se correlacionaron con el nivel población, la altura sobre el nivel del mar y la presencia de actividades de minería y vertimiento de residuos sólidos y líquidos de actividades humanas en un análisis de componentes principales (PCA) empleando el paquete estadístico Statgraphics Centurion XVII. Asimismo, se utilizaron gráficos de dispersión para analizar la relación entre las variables fisicoquímicas, biológicas y sociales con la altura y el nivel de población.

Los datos incluidos corresponden a valores promedio obtenidos en muestreos y levantamientos en campo, realizados entre 2009 y 2014, en el marco de caracterizaciones de ecosistemas estratégicos desarrolladas por el IIAP. Las mediciones fueron realizadas en estaciones de muestreo establecidas en las zonas de nacimiento de los ríos Cucurrupí y Copomá localizadas en la vertiente occidental del páramo de Duende y el río Ingará en el páramo Tatamá en el área correspondiente al departamento del Chocó; en la zona alta de la quebrada Chocolate ubicada en el cerro de Chageradó en el área de influencia del resguardo indígena Lano, 


\section{Bioetnia Volumen 12, 2015}

en el municipio de Frontino, Antioquia. Para el caso de los ríos Timbiquí y Bubuey se ubicaron estaciones en la zona media de la cuenca, la cual tiene influencia de comunidades negras e indígenas de la Organización Zona Baja Eperara Siapidaara-Cabildos y Autoridades tradicionales del Cauca (OSBEZCAC); también se tuvo en cuenta la zona media y baja del río Quito en Quibdó, Chocó y específicamente en el río Tutunendo, se localizaron estaciones desde la zona alta hasta la desembocadura. Finalmente, para el caso de los ríos Jella, Dagua, la quebrada Huina y el caño Waffe se realizaron mediciones en el área de interconexión con la zona costera de Bahía Solano, Buenaventura y Turbo respectivamente.

\section{Resultados y discusión}

El análisis agrupó los registros en cinco componentes que en conjunto explican el $90 \%$ de la variabilidad de los datos, lo cual permitió la reducción de las 17 variables evaluadas (Figura 1 y Tabla 1).

El primer componente agrupa los fosfatos, sulfatos y nitratos con el número de individuos de macroinvertebrados acuáticos, mostrando que la abundancia de este grupo biológico a lo largo de la región responde a la concentración de nutrientes en el agua, los cuales a su vez están relacionados con las dinámicas naturales del medio que pueden sufrir alteraciones bruscas como respuesta a fe-

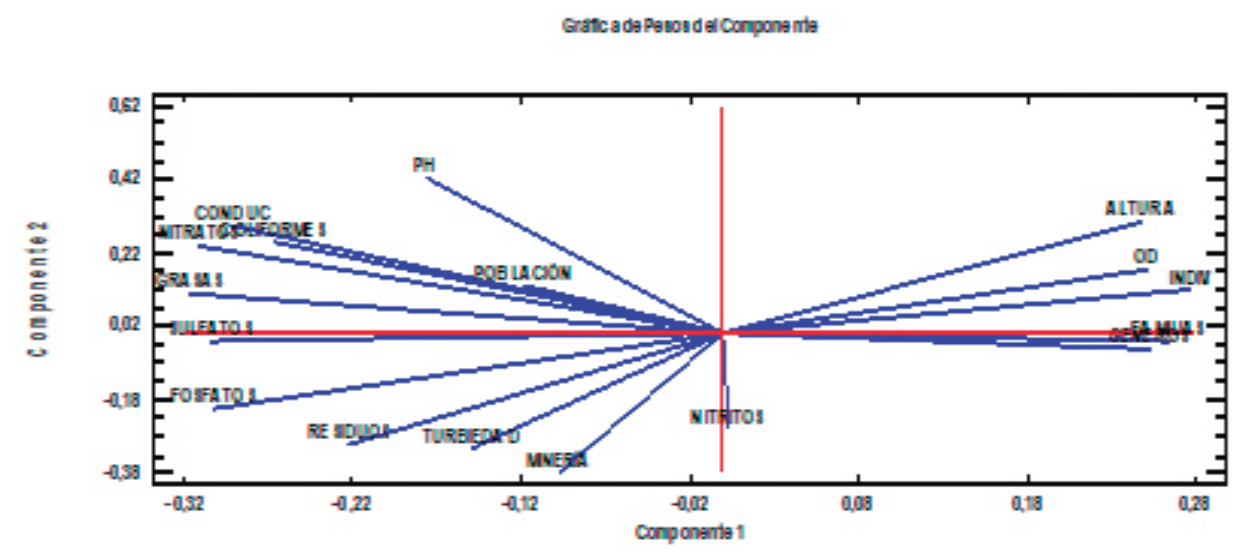

Figura 1. Análisis de componentes principales de las variables

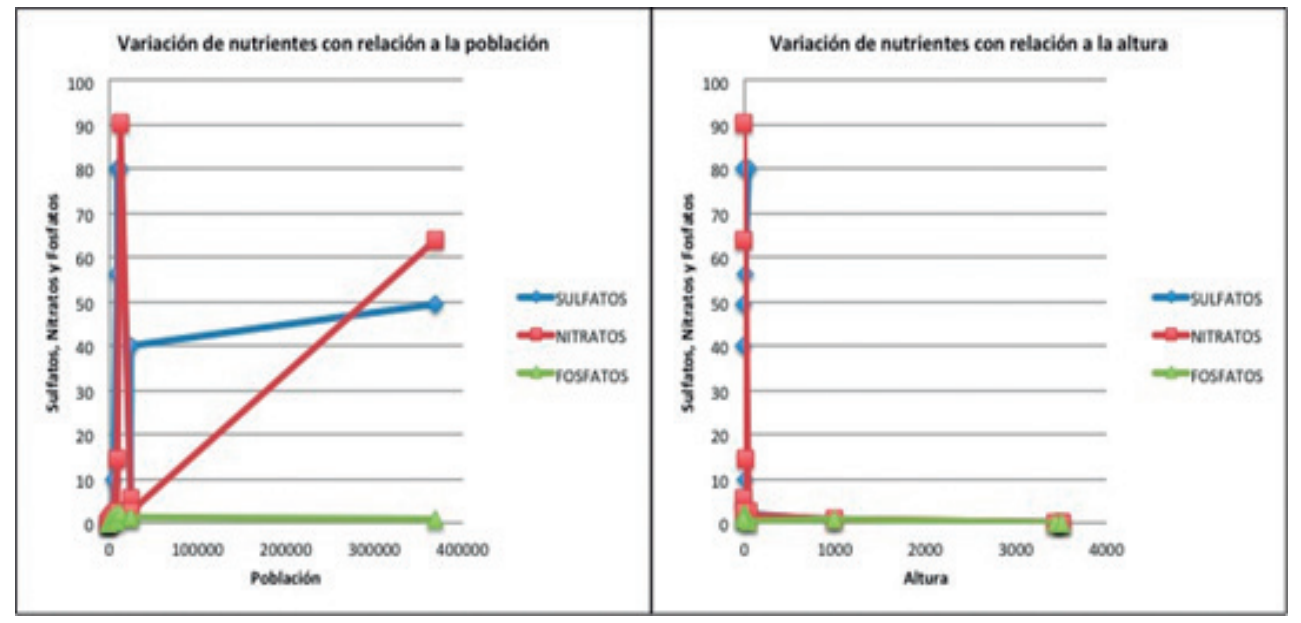

Figura 2. Relación de nutrientes vs. altura y población 
Calidad del agua y ocupación de territorio. L Vargas Porras

Tabla 1. Peso de los componentes

\begin{tabular}{lrrrrr}
\hline & \multicolumn{5}{c}{ Componente } \\
\cline { 2 - 6 } & $\mathbf{1}$ & $\mathbf{2}$ & $\mathbf{3}$ & $\mathbf{4}$ & \multicolumn{1}{c}{$\mathbf{5}$} \\
\hline Altura & 0,274204 & 0,269551 & $-0,280359$ & $-0,0531523$ & 0,26831 \\
Coliformes & $-0,250173$ & 0,206127 & $-0,168503$ & 0,422874 & 0,0749259 \\
\hline Conductividad & $-0,103813$ & 0,247575 & 0,482521 & $-0,292604$ & 0,152999 \\
\hline Familias & 0,279921 & $-0,111877$ & 0,29233 & 0,329844 & 0,0485602 \\
\hline Fosfatos & $-0,322267$ & $-0,196759$ & $-0,112576$ & 0,067392 & $-0,00644264$ \\
\hline Géneros & 0,268572 & $-0,133236$ & 0,290862 & 0,352764 & 0,0621342 \\
\hline Grasas & $-0,311764$ & 0,078287 & $-0,136775$ & 0,361994 & 0,129639 \\
\hline Individuos & 0,300747 & 0,0449506 & 0,0462484 & 0,205441 & 0,122158 \\
\hline Minería & $-0,127021$ & $-0,350019$ & $-0,16512$ & $-0,265294$ & 0,302344 \\
\hline Nitratos & $-0,301108$ & 0,273586 & 0,135369 & 0,1654 & 0,219079 \\
\hline Nitritos & $-0,00316971$ & $-0,320761$ & 0,287832 & 0,286784 & 0,356754 \\
\hline Od & 0,272084 & 0,14037 & $-0,0771305$ & $-0,0282426$ & 0,386875 \\
\hline Ph & $-0,152817$ & 0,449792 & 0,0022593 & 0,152677 & 0,101101 \\
\hline Población & $-0,120643$ & 0,236588 & 0,477945 & $-0,2984$ & 0,138831 \\
\hline Residuos & $-0,247382$ & $-0,279744$ & 0,293506 & 0,0839826 & $-0,265959$ \\
\hline Sulfatos & $-0,315426$ & 0,0224825 & 0,0311056 & 0,048975 & $-0,0022371$ \\
\hline Turbiedad & $-0,173266$ & $-0,299042$ & $-0,0816527$ & $-0,138286$ & 0,586865 \\
\hline
\end{tabular}

nómenos antrópicos que pueden incrementar sus niveles en el agua. Al respecto, algunos autores como Pacheco y Cabrera (2003) indican que los nutrientes en las aguas superficiales se encuentran en pequeñas concentraciones y se derivan de la descomposición natural de materiales por acción de microorganismos. Sin embargo, actualmente es claro que los niveles de estos elementos en los cuerpos de agua se incrementan como resultado de vertimientos líquidos y sólidos de origen antrópico, de ahí que el comportamiento de las concentraciones de estos nutrientes a lo largo de la región varíen proporcionalmente con el nivel poblacional (Figura 2). En este sentido, Moreau et al. (1998) expresan que existe una fuerte correlación entre las cargas de nitrógeno y fósforo total en los ríos con el uso de la tierra y en especial con las prácticas agrícolas. Históricamente la carga de nutrientes en los ríos ha ido aumentando de forma paralela al incremento de poblaciones humanas en sus cuencas, como resultado tanto de las aguas residuales provenientes de los aportes humanos como de la de animales y al aumento de la aplicación de fertilizantes en las tierras de cultivo, con la consiguiente lixiviación a los ríos (Cooper, 1995).

Lo anterior explica también el hecho de que en la región del Chocó Biogeográfico las mayores concentraciones de nutrientes en los ríos se hayan reportado para las zonas más bajas (Figura 2), porque en ellas se localiza la mayor cantidad de población y con ello de prácticas productivas que generen dichos incrementos en los aportes de nutrientes al agua, lo que a su vez está relacionado con las características culturales de las comunidades que habitan el territorio, que no suelen ocupar áreas por encima de los 1.000 msnm, ni para establecer asentamientos o centros poblados, ni para el aprovechamiento de recursos naturales en actividades productivas, a diferencia de otras regiones del país donde las zonas de media y alta montaña han sido colonizadas por poblaciones, actividades agrícolas, ganadería, establecimiento de bosques para producción de 


\section{Bioetnia Volumen 12, 2015}

papel e incluso minería. Mena y Hofstede (2006) y Ramón (2009) han indicado que en todos los páramos latinoamericanos, durante el siglo XIX y comienzos del XX, el proceso de poblamiento $\mathrm{y}$ apropiación del territorio, iniciado durante la colonia, desembocó en la intensificación de las actividades productivas y extractivas de productos forestales maderables y no maderables, como leña, maderas, plantas medicinales, tintes, resinas y gomas y adicionalmente minerales, como galena, carbón y sal. Para Colombia se ha reportado que a partir del año 1821, los grupos indígenas de las zonas andinas fueron agrupados en resguardos, iniciándose el proceso de colonización del páramo y unido a ello, la marginación social de la población rural e indígena y la sobreexplotación del ecosistema (Friede, 1976; Bernsen, 1991; Triana, 1992). Es así que los procesos de transformación del páramo han sido constantes, siendo en algunos momentos mucho más intensos los cambios, como en el caso del período moderno; en él, se aprecia, la incidencia antrópica sobre estos ecosistemas (Baca, 2014).

En relación con lo anterior, Marten (2001) ha indicado que en los páramos, como en los demás ecosistemas, la agricultura pasó del policultivo al monocultivo y se hizo más independiente del medio ambiente, mediante el riego, los fertilizantes químicos y los plaguicidas, lo cual se hizo posible gracias a la innovación tecnológica y a la energía proveniente de los combustibles fósiles. En la mayoría de estos ecosistemas, se desarrollan actividades mineras, forestación con especies introducidas y construcción de vías, acueductos, sistemas de drenaje y represas (Hofstede et al., 2002; Young, 1994). Actualmente, en zonas paramunas existen comunidades indígenas y campesinas asentadas, que practican la agricultura a pequeña escala, la ganadería bovina y ovina y quemas asociadas (Baca, 2014), situación contraria a lo que ocurre en los páramos y ecosistemas de media montaña de la cordillera occidental de la región del Chocó Biogeográfico, donde por cultura las comunidades no habitan estas zonas $y$ en algunos casos son considerados sitios sagrados o áreas de gran importancia con las que se conectan a través de los ríos que nacen en estos ambientes. Este comportamiento y connotación sociocultural de los habitantes de la región ha permitido la conservación tanto del ecosistema como de la calidad del agua.

Esta situación se ve reflejada en el número de individuos de macroinvertebrados que se reportaron en las zonas evaluadas, que fue mayor en las zonas altas donde se presentaron rangos normales en la concentración de estos nutrientes, a diferencia de las zonas bajas donde además de evidenciarse su incremento consecuente con el aumento de la población, se presenta una notable reducción no solo en el número de individuos sino también en la diversidad de los mismos, representada en el número de géneros y familias, las cuales presentaron reportes más bajos a menores alturas, lo que puede estar relacionado con que la degradación de la materia orgánica proveniente de las actividades humanas que se concentran en estas zonas causa una disminución del $\mathrm{pH}$ y del oxígeno disuelto y un aumento de los niveles de nutrientes como nitrógeno (Winkler, 1999; Timm et al., 2001; Rueda et al., 2002). Este tipo de comportamiento de aumento en la abundancia de macroinvertebrados con niveles moderados o bajos de enriquecimiento orgánico o su reducción en tramos con fuerte polución han sido ya documentados en trabajos como los de Prenda y Gallardo (1996), Thorne y Williams (1997) en los que se ha estudiado la respuesta de este grupo biológico ante fenómenos de contaminación del agua. Otros autores como Arévalo et al. (2001), han expresado que la temperatura del agua es un factor que incide directamente sobre la abundancia y la diversidad de macroinvertebrados debido a la variación en el gradiente altitudinal, seguido por el ingreso de materia orgánica proveniente de actividades industriales, turísticas y domésticas, lo que corrobora el patrón de comportamiento de las 
variables analizadas en este primer componente para la región del Chocó Biogeográfico.

El análisis arrojó un segundo componente que agrupa la altura, la conductividad, la presencia de actividad minera, la generación de residuos y el $\mathrm{pH}$ del recurso hídrico, indicando la existencia de un comportamiento similar entre estas variables, lo que puede obedecer a distintos factores. Uno de ellos es que las técnicas de extracción utilizadas en la región están basadas en la remoción de las capas de suelo para su posterior lavado con agua a presión, que es reincorporada de manera directa a las fuentes hídricas que son empleadas para este tipo de explotación, lo cual puede tener un efecto directo en el nivel del $\mathrm{pH}$ del agua debido a la dilución de los iones contenidos en los sustratos removidos. De este modo, aunque en términos generales el $\mathrm{pH}$ en aguas naturales puede variar en un amplio rango de valores, Fuentes y Massol (2002) indican que esta variación depende de factores intrínsecos y extrínsecos al ambiente acuático como la composición de suelos adyacentes, depósitos superficiales y lecho rocoso, así como el drenaje de minas, lo que explica el resultado obtenido, el cual permitiría inferir además que la actividad minera y sus técnicas de extracción en la región del Chocó Biogeográfico pueden ser un criterio que modela el patrón de comportamiento del pHen las fuentes hídricas y específicamente en aquellas que son destinadas a la extracción de minerales. Esta actividad junto con el vertimiento de residuos sólidos y líquidos a los cauces naturales, tienen una relación directa con la conductividad del agua, lo que demuestra un patrón esperado altitudinalmente, que da cuenta de la respuesta que tiene el aumento del nivel poblacional con la generación de desechos y su aporte de materiales que incrementan la concentración de sustancias disueltas, las cuales son responsables del aumento en la conductividad del agua.

La influencia marina juega un papel importante en el incremento de esta variable en las zonas costeras, debido a la salinidad aportada por el mar, de ahí que los niveles más altos de conductividad se hayan reportado para las zonas de interconexión costera en los ríos Jella, Huina, Dagua y el caño Waffe; sin embargo, cabe mencionar que estos dos últimos presentaron niveles muy superiores que alcanzaron hasta $18.824,25$ $\mu \mathrm{S} / \mathrm{cm}$, por la gran diferencia en aporte de residuos sólidos y aguas servidas con Jella y Huina donde el valor máximo solo alcanzó los 436,5 $\mu \mathrm{S} / \mathrm{cm}$, situación que corrobora lo afirmado por Fuentes y Massol (2002) acerca del incremento que ejerce el vertimiento de desechos y aguas residuales sin tratamiento sobre la conductividad y el deterioro de la calidad de las aguas superficiales. Este panorama muy común a lo largo de la región, donde una gran parte de los centros poblados carece de sistemas de saneamiento básico que garanticen una gestión ambiental adecuada de los residuos sólidos y el tratamiento de las aguas residuales antes de ser vertidas a los ecosistemas acuáticos, lo cual constituye un factor que determina el comportamiento y el estado del recurso.

En el tercer componente se relacionaron las variables número de familias y géneros de macroinvertebrados con la concentración de nitritos, mostrando nuevamente que la presencia y diversidad de este grupo biológico depende estrictamente de aspectos como la concentración de nutrientes, que responde a su vez al nivel y tipo de intervención antrópica. Para este caso es necesario indicar que según autores como Gray (1997), Pardo y Marañón (1997), y Prat et al. (1999), por lo general la concentración de los nitritos en el agua superficial es muy baja, pero puede presentar niveles inesperadamente altos debido a la contaminación industrial o de aguas residuales domésticas, lo que explica que esta variable presenta un comportamiento proporcional al nivel poblacional e inverso a la diversidad de macroinvertebrados, que arrojó sus valores más altos en zonas poco o medianamente intervenidas entre las que se pueden mencionar los ríos Cucurrupí en el páramo del Duende, la quebrada Chocolate en el cerro Chageradó y el 


\section{Bioetnia Volumen 12, 2015}

río Tutunendo en la selva pluvial central chocoana. Esto se explica con los efectos negativos que representan los nitritos para el desarrollo de la vida en los ecosistemas acuáticos. $\mathrm{Al}$ respecto Erikson (1985) indica que valores entre 0.1 y 0.9 $\mathrm{mg} / \mathrm{l}$ pueden presentar problemas de toxicidad dependiendo del $\mathrm{pH}$ y Prat et al. (1996), afirman que valores por encima de $1 \mathrm{mg} / \mathrm{l}$ representan un impedimento para el desarrollo de la vida de peces $y$ el establecimiento de un ecosistema fluvial en buenas condiciones.

El siguiente componente agrupó las variables población, coliformes fecales, grasas y aceites, comportamiento que obedeció a que este tipo de contaminantes en el agua son exclusivamente aportados por actividades antrópicas tanto domésticas como industriales, de ahí que sus valores hayan aumentado proporcionalmente a la distribución de la población en el territorio, la cual se encuentra concentrada en las zonas bajas. De este modo los reportes más altos tanto para coliformes como para grasas y aceites se encontraron en las quebradas Chinchano y Agua Dulce en Timbiquí, río Quito, Jella, Huina, Dagua y el Waffe en Turbo, donde además de localizarse centros poblados con deficiencias en los sistemas de saneamiento básico que garanticen la reducción de coliformes fecales mediante el tratamiento de los efluentes de alcantarillado, se ubican de manera combinada actividades turísticas y portuarias que son altamente aportantes de grasas y aceites a los cuerpos de agua, afectando su calidad.

En relación con este resultado, se puede concluir que la mayor concentración de población en ciertas zonas donde además se presentan deficiencias en la cobertura y eficiencia de los sistemas de saneamiento básico, genera mayor presión sobre los recursos hídricos y mayor contaminación de origen doméstico. Sloto (1987) y Weaver y Garman (1994) han expresado que la combinación de un rápido crecimiento poblacional, asociada con la industrialización y la urbanización, ha provocado un paulatino aumento de las presiones sobre los recursos hídricos de diferentes países. La urbanización de las cuencas provoca cambios en el uso del suelo, pasando de áreas rurales a áreas residenciales o industriales, lo que incrementa los vertidos urbanos, acumula sustancias tóxicas y aumenta la demanda de agua, reduciendo su calidad y aumentando la presencia de agentes patógenes y residuos de grasas.

Por otro lado, un último componente relacionó la disponibilidad de oxígeno disuelto y la turbiedad del agua, variables que presentan una proporción inversa teniendo en cuenta que los altos niveles de turbidez están asociados con la presencia de partículas suspendidas en el agua tales como sedimentos, efluentes residuales y plancton, lo que tiene un efecto directo en la concentración de oxígeno disuelto porque las partículas sólidas impiden el paso de la luz solar, bloqueando la fotosíntesis de las plantas acuáticas, haciendo que produzcan menos oxígeno y con ello reduciendo su disponibilidad en el agua.

De manera general el análisis descriptivo de las variables mostró una gran dispersión en los datos relacionados con la concentración de sustancias disueltas o suspendidas en el agua; de este modo, variables como turbiedad, conductividad, nutrientes, grasas y coliformes fecales, presentaron las mayores desviaciones en el comportamiento de los datos (Tabla 2), lo que está relacionado de manera directa con una gran disparidad en las concentraciones de estas variables entre las zonas analizadas, porque se presentaron valores bajos o nulos en las zonas de mayor altitud frente a niveles muy elevados en las zonas bajas y costeras. En contraste, variables como $\mathrm{OD}, \mathrm{pH}$, número de familias y géneros de macroinvertebrados, mostraron una menor desviación en sus datos, indicando que a pesar de que existen diferencias en su distribución a nivel altitudinal y poblacional, presentan una menor diferencia en el rango de variación. 
Calidad del agua y ocupación de territorio. L Vargas Porras

Tabla 2. Valores de estadísticos de tendencia central de las variables

\begin{tabular}{|lrrr}
\hline Variables & Desviación & Promedio & Varianza \\
\hline Altura & 1546,23 & 962,583 & $2,39 \mathrm{E}+06$ \\
\hline Minería & 0,389249 & 0,166667 & 0,151515 \\
\hline Residuos & 0,452267 & 0,75 & 0,204545 \\
\hline Población & 104840 & 37953,1 & $1,10 \mathrm{E}+10$ \\
\hline Od & 2,52195 & 5,81833 & 6,36023 \\
\hline Ph & 0,624014 & 6,46708 & 0,389393 \\
\hline Conduct. & 3803,99 & 1151,37 & $1,45 \mathrm{E}+07$ \\
\hline Turbiedad & 137,862 & 62,7667 & 19005,8 \\
\hline Sulfatos & 32,258 & 26,9042 & 1040,58 \\
\hline Nitratos & 29,6681 & 15,3017 & 880,198 \\
\hline Nitritos & 0,422183 & 0,215417 & 0,178239 \\
\hline Fosfatos & 0,813406 & 0,953333 & 0,661629 \\
\hline Coliformes fecales & $6,71 \mathrm{E}+07$ & $1,94 \mathrm{E}+07$ & $4,51 \mathrm{E}+15$ \\
\hline Grasas y a & 20,0022 & 12,4725 & 400,088 \\
\hline$N^{\circ}$ de individuos & 47,8187 & 39,0833 & 2286,63 \\
\hline Familias & 6,35324 & 7 & 40,3636 \\
\hline Géneros & 8,66375 & 8,83333 & 75,0606 \\
\hline
\end{tabular}

\section{Conclusiones}

El análisis permitió concluir fuertes relaciones entre la concentración de nutrientes y el número de individuos de macroinvertebrados; además, entre la altitud, la conductividad, el $\mathrm{pH}$ y la presencia de vertimientos mineros y desechos antrópicos, así como también entre el nivel poblacional y la concentración de coliformes fecales, grasas y aceites, mostrando que el patrón de comportamiento de la calidad de agua en la región está determinado no solo por el nivel poblacional presente en el área de influencia de las fuentes hídricas, sino por una combinación de elementos que incluyen el tipo de prácticas productivas desarrolladas, el estado de los sistemas de saneamiento básico y la distribución altitudinal de la ocupación del territorio que es una característica cultural de la región.

\section{Literatura citada}

Arévalo JE, Serrato C, Velez GA, Gaviria L. 2001. Ma- croinvertebrados acuáticos y su relación con nutrientes presentes en las cuencas de los ríos Guacimaly San Luis, Costa Rica. San José: Asociación Conservacionista de Monteverde. URL disponible en: http://www.eco-index.org/search/results_es.cfm?projectID $=1052 \#$. WdT2gGjWyUk

Baca-G AE. 2014. Reflexiones sobre los procesos de ocupación humana en los páramos. Situación actual del páramo volcán Chiles, Colombia. Rev UDCAAct \& Div Cient. 17(1): 217-26. URL disponible en: http://www. udca.edu.co/wp-content/uploads/2014/12/reflexiones-sobre-procesos-ocupacion-humana-paramos-situacion-actual-paramo-volcan-chiles-colombia.pdf

Bernsen O. 1991. Observaciones preliminares sobre el cultivo en zonas de páramo de Colombia. Nov Col. 3: 63-73.

Cooper SR. 1995. Chesapeake Bay watershed historical land use: impact on water quality and diatom communities. Ecol Applic. 5 (3): 703-23. URL disponible en: https://www.jstor.org/stable/1941979?seq=1\#page scan tab contents

Coto J. 1998. Calidad de las aguas del río Virilla, quebradas y tributarios, en diversos puntos ubicados en la cuenca alta del Virilla. Revista CNFL (PLAMA-VIRILLA). 7: 38-50.

Erikson RJ. 1985. An evaluation of mathematical models for 


\section{Bioetnia Volumen 12, 2015}

the effects of $\mathrm{pH}$ and temperature on ammonia toxicity to aquatic organism. Water Res. 19 (8): 1047-58. URL disponible en: http://www.sciencedirect.com/science/ article/pii/0043135485903756

Friede J. 1976. El indio en la lucha por la tierra. Tercera edición ampliada y revisada. Bogotá: Punta de Lanza; 25 pp.

Fuentes F, MassolA. 2002. Manual de ecología microbiana. Ecología de microorganismos. Mayagüez: Universidad de Puerto Rico; 265 pp. URL disponible en: http://www. uprm.edu/biology/profs/massol/manual/p1-intro.pdf

Gray NF. 1997. Calidad del agua potable. Problemas y soluciones. Zaragoza: Editotial Acribia SA. 365 pp.

Hofstede R, Coppus R, Mena P, Segarra P, Wodlf J, Sevink J. 2002. El estado de conservación de los páramos de Pajonal en el Ecuador. Ecotropicos. 15 (1): 3-18. URL disponible en: http://www.portalces.org/sites/default/ files/references $/ 037$ Hofstede $\% 20$ et $\% 20 \mathrm{al} \% 20$ ecotropicos.2002.pdf

Marten G. 2001. Ecología humana: Conceptos básicos para el desarrollo sustentable. Londres: Earthscan Publications; 256 pp. URL disponible en: http://www. gerrymarten.com/ecologia-humana/capitulo01.html

Mena P, Hofstede R. 2006. Los páramos ecuatorianos. En: Moraes M. Øllgaard B, Kvist LP, Borchsenius F, Balslev H (eds.). Botánica económica de los Andes Centrales. La Paz: Universidad Mayor de San Andrés; p. 91-109. URL disponible en: http://www.beisa.dk/Publications/ BEISA $\% 20$ Book $\% 20$ pdfer $/ 1 \% 20$ Indice.pdf

Moreau S, Bertru G, Buson C. 1998. Seasonal and spatial trends in nitrogen and phosphorus loads to the upper catchment of the river Valaine (Brittany): relationships with land use. Hydrobiologia. 373: 247-58. URL disponible en: https://link.springer.com/article/10.1023/A:1017031907740

Pacheco J, Cabrera A. 2003. Fuentes principales de nitrógeno de nitratos en aguas subterráneas. Ingeniería. 7 (2): 47-54. URL disponible en: http://www.redalyc. org/articulo.oa?id=46770204

Pardo F, Marañon E. 1997. Contaminación oceánica de las plantas. En: Bueno JL, Sastre H, Lavin SG (eds.). Contaminación e ingeniería ambiental. Oviedo: FICYT; pp 154-88.

Prat N, Rieradevall M, Munné A, Chacón G. 1966. La qualitat ecològica de les aigües del Besòs i el Llobregat. Estudis de la qualitat ecològica dels rius. Vol 1. Diputació de Barcelona: Àrea Medi Ambient; 102 pp.

Prat N, Rieradevall M, Munné A, Solá C, Chacón G. 1999. La qualitat ecològica de les aigües del Besós i el Llobregat. Estudis de la qualitat ecològica dels rius. Vol. 2. Àrea Medi Ambient. Diputació de Barcelona; 153 pp.

Prenda J, Gallardo-Mayenco A. 1996. Self-purification, temporal variability and the macroinvertebrate commu- nity in small lowland Mediterranean streams receiving crude domestic sewage effluents. Arch Hydrobiol. 136 (2): 159-70. URL disponible en: https://scholar.google.

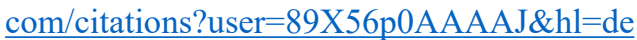

Ramón, G. 2009. Conocimiento y prácticas ancestrales. En: Mena Vásconez P, Morales M, Ortiz P, Ramón G, Rivadeneira S, Suárez E, et al. (eds). Gente y ambiente de Páramo: realidades y perspectivas en el Ecuador. Quito: Proyecto Páramo Andino; pp.13-22.

Rodríguez C, Mancini M, Prósperi C, Weyers A, Alcantú G, Ferrero S. 2002. Variaciones estacionales de la calidad del agua del río Chocancharava (río Cuarto), Córdoba, Argentina. Ecol Austral. 12 (1): 65-72. URL disponible en:http://www.scielo.org.ar/scielo.php?script=sci arttext\&pid=S1667-782X2002000100009

Rueda J, Camacho A, Mézquita F, Hernández R, Roca JR. 2002. Effect of episodic and regular sewage discharges on the water chemistry and macroinvertebrate fauna of a Mediterranean stream. Water, Air and Soil Pollution, 140 (1-4): 425-44. URL disponible en: https://link. springer.com/article/10.1023/A:1020190227581

Sloto RA. 1987. Effect of urbanization on the water resources of eastern Chester County, Pennsylvania. US Geological Survey. Water-Resources Investigations Report 87-4098; 131 pp. URL disponible en: https:// pubs.usgs.gov/wri/1987/4098/report.pdf

Thorne R, Williams W. 1997. The response of benthic macroinvertebrates to pollution in developing countries: a multimetric system of bioassessment. Freshwater Biol. 37(3): 671-86. URL disponible en: http://onlinelibrary. wiley.com/doi/10.1046/j.1365-2427.1997.00181.x/ $\underline{\text { abstract }}$

Timm H, Ivask M, Möls T. 2001. Response of macroinvertebrates and water quality to long-term decrease in organic pollution in some Estonian streams during 1990-1998. Hydrobiologia. 464 (1-3): 153-64. URL disponible en: https://link.springer.com/article/10.1023/A:1013999403209

Triana A. 1992. La colonización española en el Tolima: siglos XVI y XVII. Bogotá: FUNCOL; 308 pp.

Weaver LA, Garman GC. 1994. Urbanization of a watershed and historical changes in a stream fish assemblage. Trans Am Fish Soc. 123: 162-72.

Winkler MA. 1999. Tratamiento biológico de las aguas de desecho. Ciudad de México: Limusa Noriega Editores; 338 pp. URL disponible en: http:/www.teorema.com. $\mathrm{mx} /$ tendencias/tratamiento-biologico-de-aguas-de-desecho/

Young KR. 1994. Roads and the environmental degradation of tropical montane forest. Conserv Biol. 8 (4): 972-6. http://onlinelibrary.wiley.com/doi/10.1046/j.15231739.1994.08040972.x/full 